\title{
Numerical simulation of Joule-heating flow in a cuboid cavity by the GSMAC method
}

\author{
J. Zhou ${ }^{1}$, N. Tsuzuki ${ }^{2} \&$ H. Kikura ${ }^{1}$ \\ ${ }^{1}$ Department of Nuclear Engineering, \\ Tokyo Institute of Technology, Japan \\ ${ }^{2}$ The Institute of Applied Energy, Japan
}

\begin{abstract}
Flow behaviour was observed in simplified model cavities of a high level liquid waste (HHLW) reprocessing glass melter. The effect of heating volume on the Joule-heating flow was evaluated using two cavities with different heating volumes. Electrodes were set to generate Joule-heating. A chaotic flow occurred because the lower part of the cavities was heated while the top surface of the cavities was cooled. Downflow and upflow occurred alternately in cavities. One cavity was a simple cubic cavity, for which it was easy to observe effects of thermal boundary conditions on Joule-heating flow. The other one was a cuboid cavity, which has the same aspect ratio of the upper part of the glass melter. Jouleheating flow behaviour in cavities was calculated by the GSMAC-FEM (Generalized Simplified Marker and Cell-Finite Element Method) code. The GSMAC method can calculate flow field, thermal field and electro-magnetic field as a fully coupled analysis, which exists in the glass melter. To know the flow behaviour in each cavity for the condition with electrode cooling and the condition without electrode cooling, 1-D flow behaviour and 2-D flow behaviour were calculated. It was found that the chaotic flow behaviours generated by Jouleheating were changed by the different conditions. A stable upflow occurred under the chaotic flow area in cavities for the electrode cooling condition. However, the aspect ratio decided the strength of the stable upflow. In the case of the cuboid cavity, the effect of the downflow near the electrodes decreased. The same phenomena could be predicted in the melter.

Keywords: GSMAC method, Joule-heating flow, chaotic flow, cooling condition.
\end{abstract}




\section{Introduction}

Radioactive high-level waste (HLW) is already produced all over the world as a by-product from nuclear power plants, and the method to reprocess HLW becomes an important issue to solve. In the reprocessing, HLW is dissolved and changed into High Level Liquid Waste (HLLW), and HLLW will be poured into molten borosilicate glass in a glass melter to make stable mixture of HLLW and glass for geological disposal. In Japan, a liquid fed ceramic melter (LFCM) type glass melter (Fig. 1) is being developed for the reprocessing. The glass melter applies Joule-heating to generate the molten glass, and the melter can mix HLLW and molten glass by convective flow mainly induced by Joule-heating and radiation from upper surface. These volumetric heating in lower part and cooling in the upper part make continuous chaotic flow behaviour, named as 'chaotic steady state' $[1,2]$. In fact, the chaotic flow behaviour in the glass melter is difficult to understand, and the melter operation is sometimes aborted when an accident is observed in the melter. Understanding the chaotic flow behaviour is important for effective melter operation, however, there are many effects on the flow behaviour of electrode cooling [3], cold cap [4], platinum group [5], foaming reagent [6], etc. Thus, former studies on chaotic flow behaviour are executed using a simple cubic cavity. In the case of measuring the flow behaviour of molten glass, the high temperature of molten glass makes it difficult. Therefore, CFD is applied to predict the Joule-heating flow behaviour. Aoshima [7] used CFD code FLUENT to predict flow behaviour in the glass melter.

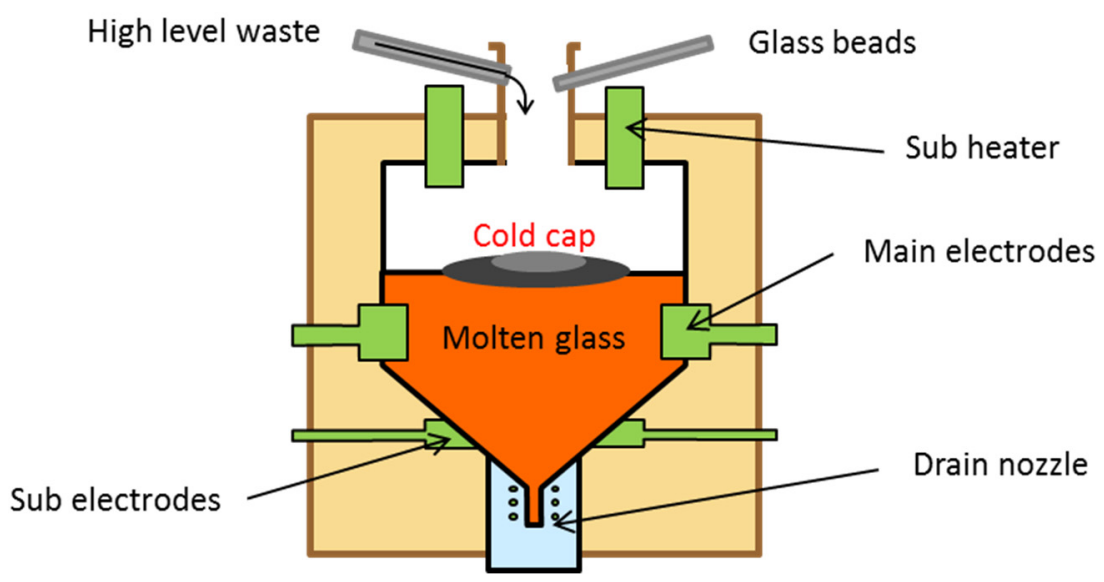

Figure 1: LFCM glass melter.

However, the previous numerical study focused on the flow field and temperature field, the magnet field was ignored. In the glass melter, the flow behaviour is considered based on the flow field, temperature field and magnet field. To developing flow analysis and fluid measurement technology of molten glass, CFD code is required to combine these three fields. GSMAC-FEM (Generalized 
Simplified Marker and Cell-Finite Element Method) code has a feature that can be cross-coupling an electromagnetic field and flow field. Duong et al. [3] is used GSMAC-FEM as a trial application for prediction of Joule-heating flow in a cubic cavity. Qualitative agreement was obtained between simulation results and experimental ones.

In addition, there are several problems of former studies by using cubic model. The most important one of the differences is the setting of the electrodes. In the cubic cavity, the electrodes were set at a pair of opposed sides. Joule-heating was carried out in whole cavity. However, the glass melter sets the electrodes on the upper parts, the Joule-heating flow just occur in the upper parts. Even though consider the cubic cavity model the upper part, the aspect ratio between glass melter and cubic cavity was different, it will lead the heat volume change. Therefore, a cuboid cavity had same aspect ratio with glass melter upper part was applied. Flow behaviour in the cuboid cavity was calculated by the GSMAC code.

\section{Numerical simulation using GSMAC}

\subsection{Governing equation}

The governing equations in original GSMAC are shown below, derived from the formulas of the velocity field, electromagnetic field and temperature field. The velocity field formula consists of the continuity equation and the Navier-Stokes equation. The electromagnetic field formula is derived from the conservation laws of current and Ohm's law, and the temperature field formula is written by using the energy equation. GSMAC executes fully-coupled analyses for the three fields (flow, thermal and electromagnetic field). More details about the calculation method in GSMAC are written in [8-10].

Equation of continuity:

Navier-Stokes equation:

$$
\nabla \cdot v=0
$$

$$
\begin{gathered}
\rho \frac{\partial v}{\partial t}+\rho(v \cdot \nabla) v=-\nabla p+\nabla \cdot(2 \mu D)+\nabla \cdot T_{e m}-\rho \beta\left(T-T_{0}\right) g \\
D=\frac{1}{2}\left\{\nabla \otimes v+(\nabla \otimes v)^{T}\right\} \\
T_{e m}=\frac{1}{2}(B \cdot H) I+B \otimes H
\end{gathered}
$$

Thermal energy equation:

$$
\begin{gathered}
\rho C_{v} \frac{\partial T}{\partial t}+\rho C_{v}(v \cdot \nabla) T=-\nabla \cdot q+2 \mu D: D+\frac{\left|J_{C}\right|^{2}}{\sigma_{e}} \\
q=-\kappa \nabla T \\
J_{C}=\sigma_{e}(E+v \times B) \\
\nabla \times\left(\frac{1}{\mu_{m}} \nabla \times A\right)=\sigma_{e}\left\{-\nabla \phi-\frac{\partial A}{\partial t}+v \times(\nabla \times A)+J_{f}\right\} \\
\nabla \cdot J_{f}=0
\end{gathered}
$$


The revision of Ampere's rule is derived from introduction of $\mathrm{A}-\varphi$ method. The magnetic vector potential $\mathrm{A}$ is introduced with the definition as:

$$
\nabla \times A=B
$$

From Gauss's law, and Faraday's law of the fundamental equations of electromagnetic field:

$$
\begin{gathered}
\nabla \cdot B=0 \\
\nabla \times E+\frac{\partial B}{\partial t}=0
\end{gathered}
$$

And then using equation (12), it will be obtained:

$$
\nabla \times\left(E+\frac{\partial A}{\partial t}\right)=0
$$

The eqn. (13) can be written as gradient of some scalar function. Thus, a scalar potential can be calculated as:

$$
\mathrm{E}+\frac{\partial A}{\partial t}=-\nabla \phi
$$

The electric current density is then derived as:

$$
J=\frac{1}{\mu_{m}} \nabla \times(\nabla \times A)=\frac{1}{\mu_{m}}\left\{\nabla(\nabla \times A)-\nabla^{2} A\right\}
$$

Using the Coulomb gauge $\nabla \times \mathrm{A}=0$, the electric current density is written as follows:

$$
\begin{gathered}
J=\frac{1}{\mu_{m}} \nabla^{2} A=J_{f}+J_{c} \\
\nabla \times J_{c}=0
\end{gathered}
$$

Using the equations (17) and (9), GSMAC-FEM solves electric current distribution.

$$
\nabla \times\left\{\sigma_{e}\left[-\nabla \phi-\frac{\partial A}{\partial t}+v \times(\nabla \times A)\right]\right\}=0
$$

\subsection{Computation method}

As a numerical method for incompressible viscous flow, we utilize the Generalized-Simplified Marker and Cell Finite Element Method (GSMAC-FEM). The energy equation and Ampere-Maxwell equation are computed by the nodebased FEM and edge based FEM, respectively. The solvers of three fields, i.e. flow field, thermal field and magnetic field, are coupled through a staggered scheme. The coupling method enables us to analyse natural convection with Joule-heating induced by an electromagnetic field.

The governing equations for the velocity field are solved by the GSMAC-FEM. The GSMAC-FEM is extended from a highly simplified marker and cell (HSMAC). The velocity and the pressure are calculated at a predictor step and a simultaneous relaxation step. The Navier-Stokes equations are discretized semi-implicitly, where the velocity and the pressure are discretized explicitly and implicitly respectively as follows:

$$
\begin{gathered}
\frac{v^{n+1}-v^{n}}{\Delta t}=-v^{n} \cdot \nabla v^{n}-\nabla p^{n+1}+v \nabla^{2} v^{n}+\nabla \cdot\left(\frac{\Delta t}{2} v^{n} v^{n}\right) \cdot \nabla v^{n}, \\
+J^{n} \times B^{n}-\beta\left(T^{n}-T_{0}\right) g
\end{gathered}
$$


where a forward difference is used for the time derivative, $\mathrm{n}$ denotes the $\mathrm{n}$-th time step, and is the time increment. Using the fractional step method, eqn (10) is divided into two parts:

$$
\begin{gathered}
\frac{\hat{v}-v^{n}}{\Delta t}=-v^{n} \cdot \nabla v^{n}-p^{n}+v \nabla^{2} v^{n}+\nabla \cdot\left(\frac{\Delta t}{2} v^{n} v^{n}\right) \cdot \nabla v^{n}, \\
+J^{n} \times B^{n}-\beta\left(T^{n}-T_{0}\right) g \\
\rho \frac{v^{n+1}-\hat{v}}{\Delta t}=-\nabla p^{c}
\end{gathered}
$$

where $\hat{v}$ is the predictor, and $\mathrm{p}^{\mathrm{c}}=\mathrm{p}^{\mathrm{n}+1}-\mathrm{p}^{\mathrm{n}}$ is the modified pressure. Eqn. (11) is solved at the predictor step. When eqn (3.1) is satisfied at the next time step, the following Poisson equation for is solved with eqn (21) at the simultaneous relaxation step:

$$
\nabla^{2} \mathrm{p}^{\mathrm{c}}=\rho \frac{\nabla \cdot \widehat{v}}{\Delta \mathrm{t}}
$$

The thermal energy equation is explicitly discretized as follows:

$$
\rho c_{v} \frac{T^{n+1}-T^{n}}{\Delta t}=-\rho C_{v}\left(v^{n+1} \cdot \nabla\right) T^{n}+\kappa \nabla^{2} T^{n}+2 \mu D: D+\frac{\left|J_{C}\right|^{2}}{\sigma_{e}}
$$

Assumptions: The above-mentioned of the governing equations have been simplified by the following assumptions:

(1) The fluid is Newtonian;

(2) The Boussinesq approximation may be applied to the buoyancy force term in eqn (19);

(3) The flow is laminar and incompressible;

(4) The displacement current is neglected.

\subsection{Numerical model}

The analytical model for Joule-heating cavity is shown in Fig. 2. Threedimensional numerical analyses of GSMAC were executed using this model. The green area is the analysis mesh of the thermoelectric ally conducting field (MHD area) and the black area is the analysis mesh of electromagnetic field. The dimension of this fluid domain is $126 \times 152 \times 76(\mathrm{~mm})$. This area is modelled by hexahedral elements with 27,000 meshes. The top surface and side wall are modelled as isothermal condition and the rest part is treated as adiabatic condition. At the initial stage, the liquid is at rest with the constant temperature $\left(20^{\circ} \mathrm{C}\right)$. The electrodes are placed on the opposite side wall of fluid domain and connected with a power source $(95 \mathrm{~V})$. To investigate the effect of the cooling condition of the electrodes plate on chaotic Joule-heating flow, the electrodes side can be changed between adiabatic and cooling by $20^{\circ} \mathrm{C}$. The electromagnetic field (black area) are modelled to cover the fluid domain which is three times bigger than the analysis mesh of the thermoelectric ally conducting field. The dimension of electromagnetic domain is $378 \times 456 \times 228(\mathrm{~mm})$ and modelled by hexahedral element with 48,000 meshes. The outer boundary of MHD region satisfies electromagnetic condition as follows:

$$
H \cdot n=0
$$

where $n$ is outward unity vector. Eq. (24) means that boundary condition for the magnetic field calculation. 


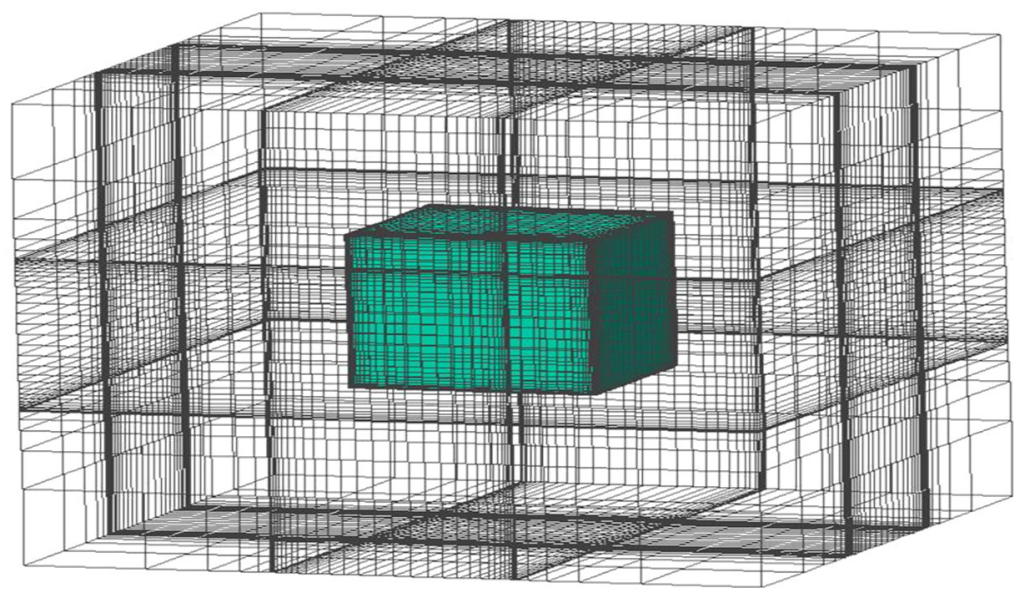

Figure 2: Calculation model and mesh layout by numerical analysis.

\section{Results and discussions}

\subsection{Temperature profile and time-dependent change in the cavity}

To know the time of flow became stable chaotic flow, time-dependent change of temperature in centre point of the cavity was observed at first. The stable temperature could be considered the flow becomes the chaotic steady state.

The temperature profile in the centre of the cavity by changing electrodes cooling condition is shown as Fig. 3. Top cooling surface is set as constant

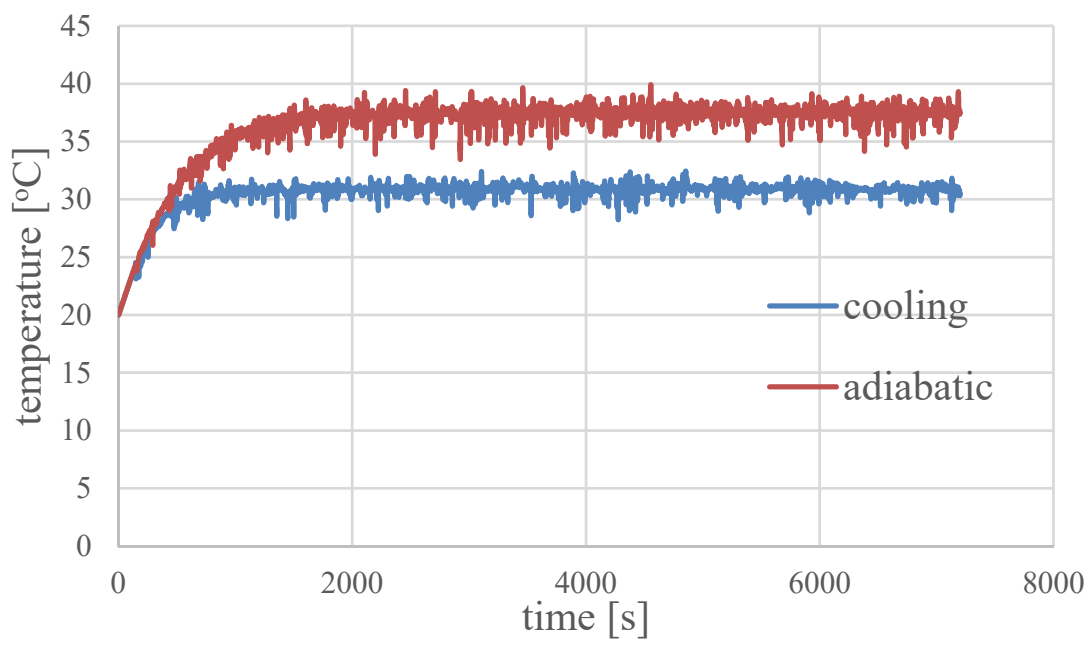

Figure 3: Time-dependent change of temperature at the centre point of the cavity. 
adiabatic and cooled at $20^{\circ} \mathrm{C}$. The Joule heating had been started with voltage between electrodes of $95 \mathrm{~V}$. The temperature response showed almost converged temperatures after sufficient time passed. In the case the electrodes surface is adiabatic, the temperature at the centre rose close to $40^{\circ} \mathrm{C}$ after $1500 \mathrm{~s}$ from the heating start. In the case of the electrodes surface is cooling, the temperature came to over than $30^{\circ} \mathrm{C}$ after around $1000 \mathrm{~s}$. Under the adiabatic case, the time of temperature became stable was later than the cooling case. It can be considered the difference of overall temperature. Because the voltage was same between 2 cases, the growth rate of temperature should be same. However, overall temperature of adiabatic condition was higher than cooling condition, therefore, the adiabatic condition need longer time to be stable. The temperature profile in the centre plate perpendicular to electrodes side can show it more obviously.

Fig. 4 shows the temperature profile in the centre plate perpendicular to electrodes at different time under 2 cases. Temperature profiles in the centre plate perpendicular to electrodes at different time are shown as Fig. 4. Figs 4(a), (c)

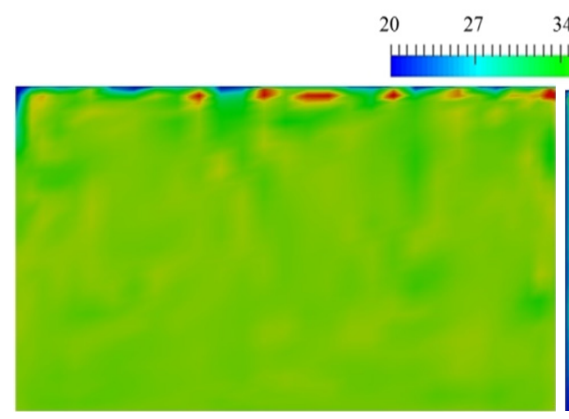

(a) $\mathrm{t}=3000 \mathrm{~s}$ adiabatic

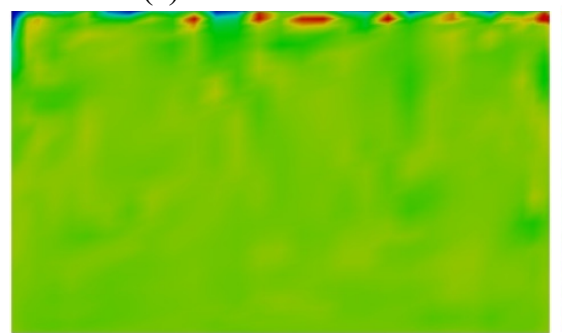

(c) $\mathrm{t}=5000 \mathrm{~s}$ adiabatic

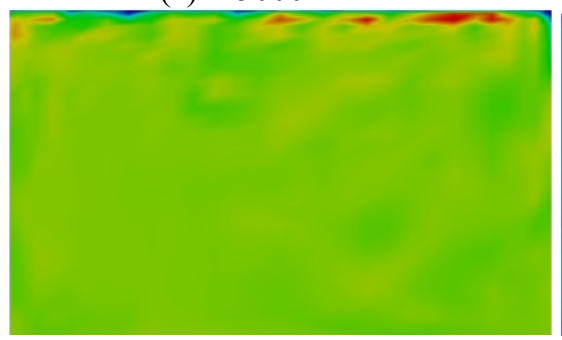

(e) $t=7000$ s adiabatic (b) $\mathrm{t}=3000 \mathrm{~s}$ cooling

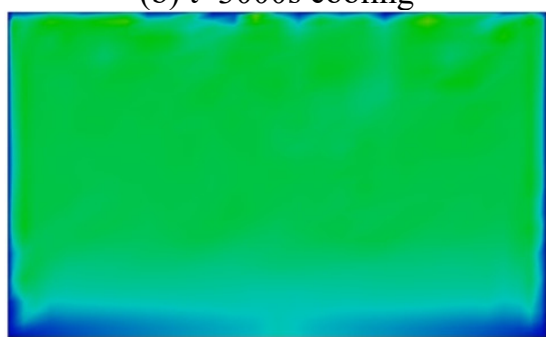

(d) $\mathrm{t}=5000 \mathrm{~s}$ cooling

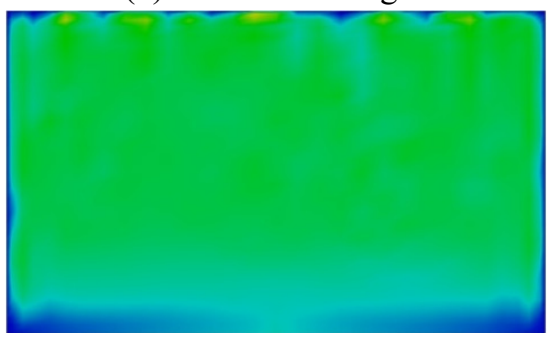

(f) $\mathrm{t}=7000$ s cooling

Figure 4: Temperature profile in the centre plate perpendicular to electrodes. 
and (e) were under adiabatic condition. Figs 4(b) (d) and (f) were under the cooling condition. The overall temperature of cavity under the adiabatic condition was higher than the cooling condition. Under the cooling condition, because the electrodes were cooled, the fluid temperature near the electrodes was lower than centre, and this cold fluid deposited in the bottom part, these cold fluid lead the overall temperature under the cooling condition was lower than the adiabatic condition.

\subsection{D velocity distribution}

Fig. 5 depicts the snapshot of the flow behaviour both cases with and without electrode cooling conditions.

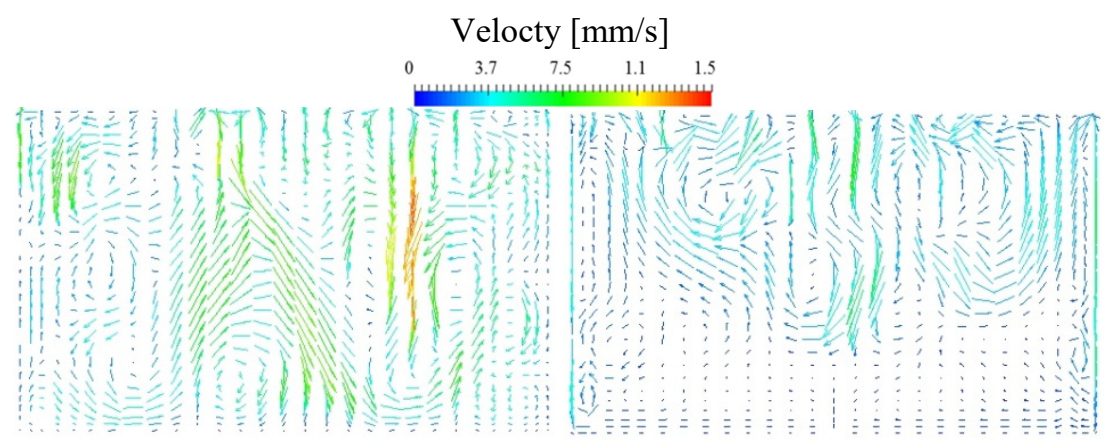

(a) $\mathrm{t}=3000 \mathrm{~s}$ adiabatic

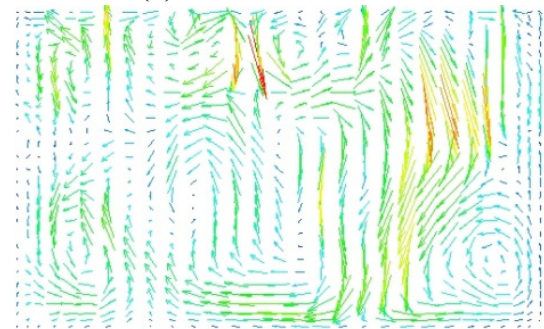

(c) $\mathrm{t}=5000 \mathrm{~s}$ adiabatic

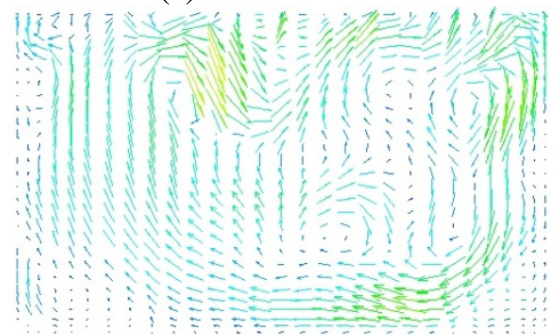

(e) $\mathrm{t}=7000 \mathrm{~s}$ adiabatic (b) $\mathrm{t}=3000 \mathrm{~s}$ cooling

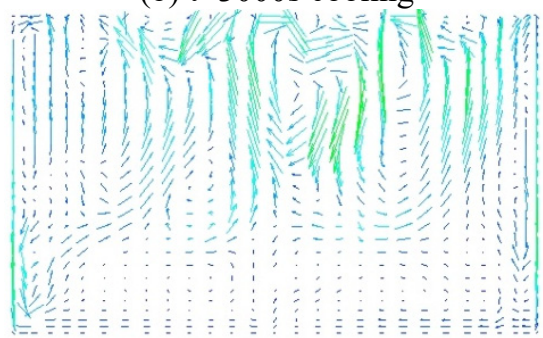

(d) $\mathrm{t}=5000 \mathrm{~s}$ cooling

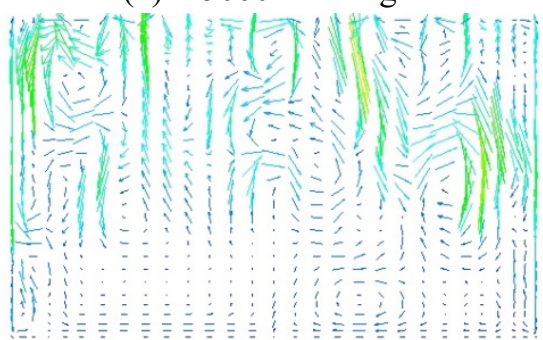

(f) $\mathrm{t}=7000 \mathrm{~s}$ cooling

Figure 5: Simulation result of velocity field. 
Figs 5(a), (c) and (e) were under adiabatic condition. Figs 5(b), (d) and (f) were under the cooling condition. The flow was unstable in whole cavity when electrode was under adiabatic condition. Meanwhile, when electrodes were cooled, the stable flow flowed to the centre can be observed in the bottom part. This stable flow flowed to the centre was the counter flow of downflow near the electrodes side. The electrodes were cooled, therefore, the temperature of flow near this area was lower than the centre, the gravity lead this cold fluid flow down, the stable downflow occurred near the electrodes side. After the downflow archived bottom of the cavity, the counter flow occurred, therefore, the flow in the bottom flowed to the centre. Therefore, the fluid temperature was low in the bottom part of the cavity like Fig. 4 has shown. This counter flow also limited the chaotic flow just occurred in the upper part of the cavity. The similar flow behaviour was also observed in the previous study [3]. The limited area of chaotic flow also means the mixing area was limited. This counter flow would weak the vitrification in the glass melter. Therefore, understanding the limited chaotic flow area is important for glass melter design.

\subsection{Velocity profile at the centre line of the cavity}

To know the limited area of chaotic flow in the cavity, the long-term flow behaviour is required because the flow changes at every position in the cavity. In order to observe the long-term flow behaviour using numerical analysis, the vertical component of velocity profile was also plotted in colour plot. Because the counter flow from 2 electrodes side would meet at the centre and became the stable upflow, the velocity was calculated at the centre line between two electrode plates at same plane of Fig. 5. Figures 6 and 7 show a (colour) plot of vertical velocity magnitude. Negative value (blue colour and green colour) indicates

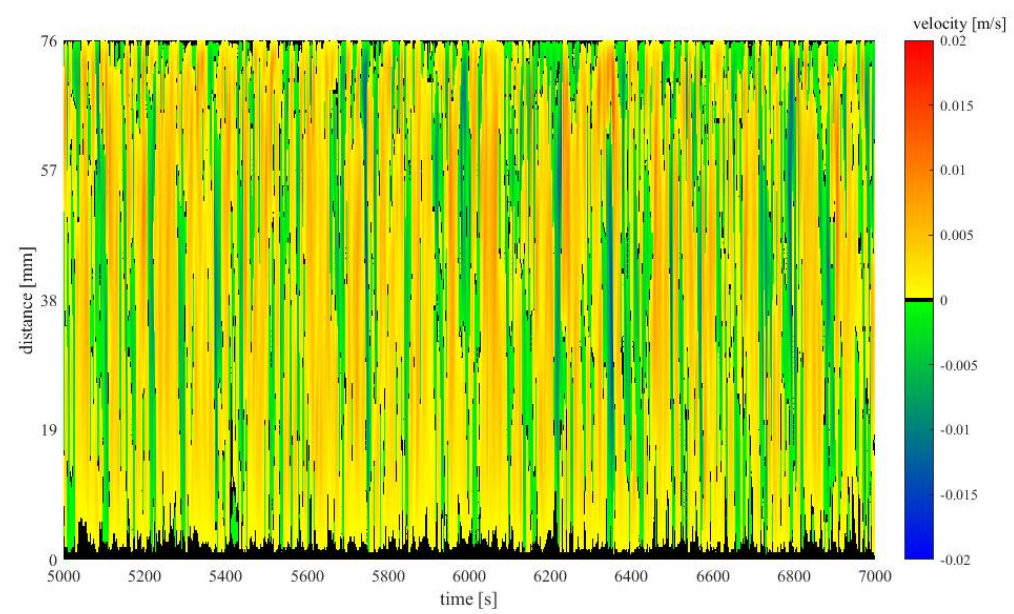

Figure 6: Vertical component of velocity profile under the electrodes were adiabatic. 
downflow meanwhile positive value (red colour and yellow colour) does upflow. The unit of flow velocity is $\mathrm{m} / \mathrm{s}$.

As a result, GSMAC could reproduce the chaotic flow behaviour in the cavity with Joule-heating. Calculation results also show the difference between the flow behaviour with electrode cooling condition and that without electrode cooling condition.

Under without electrode cooling condition, the downflow archived to the bottom of the cavity. However, if cooling the electrodes, the stable upflow was observed in the bottom of the cavity shown in Fig. 7. The chaotic flow area was limited by this stable flow.

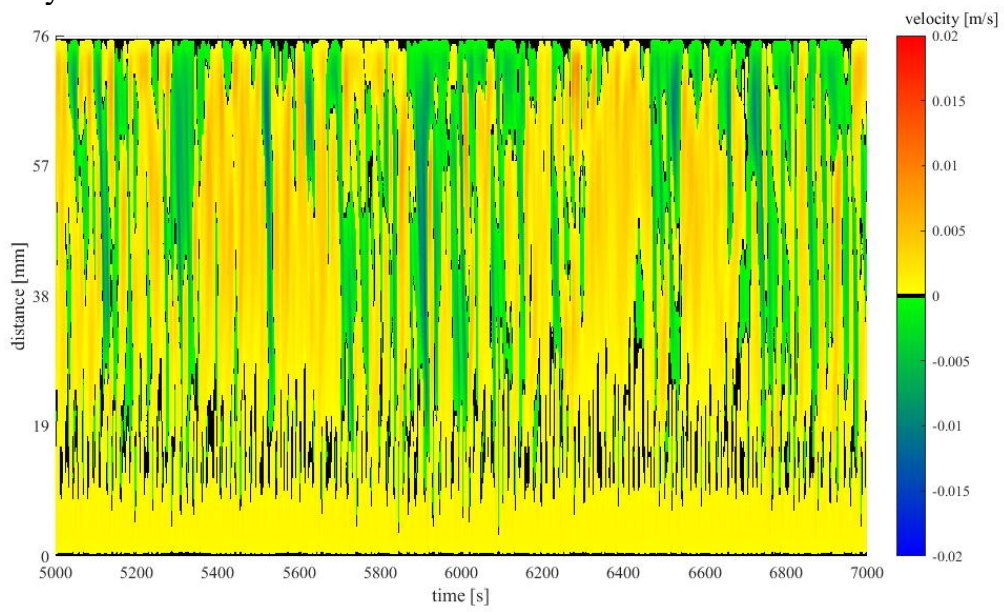

Figure 7: Vertical component of velocity profile under the electrodes were cooling.

However, the chaotic flow also can be observed in the almost whole cavity. The chaotic flow could be observed in over $80 \%$ upper part of the cavity. Fig. 8 also showed this result.

Considering the flow flowed to the bottom by the gravity, gravity direction velocity profiles of centre line at different times were shown on Fig. 8 - the same result of chaotic flow. However, in the previous study [3], the downward flow reached only around the middle of cavity. The reason could be considered the effect of aspect ratio of the cavity. The cuboid cavity has large respect ratio, the counter flow of downflow near the electrodes sides need longer time and distance to meet at the centre. Long way to the centre means more time to decrease the energy of the counter flow, therefore, in the cuboid cavity, the energy of upflow in the bottom would lower than previous case, and the upflow area was also small. The chaotic flow could be observed in over $80 \%$ upper part of the cavity. Because the upper part of the glass melter has the same respect ratio with cuboid cavity, and in the glass melter the molten glass just flows in the upper part, similar flow behaviour could be predicted. The flow in the glass melter would be occurred in the almost whole upper part of the glass melter, and the electrodes cooling condition won't weaken the vitrification. 


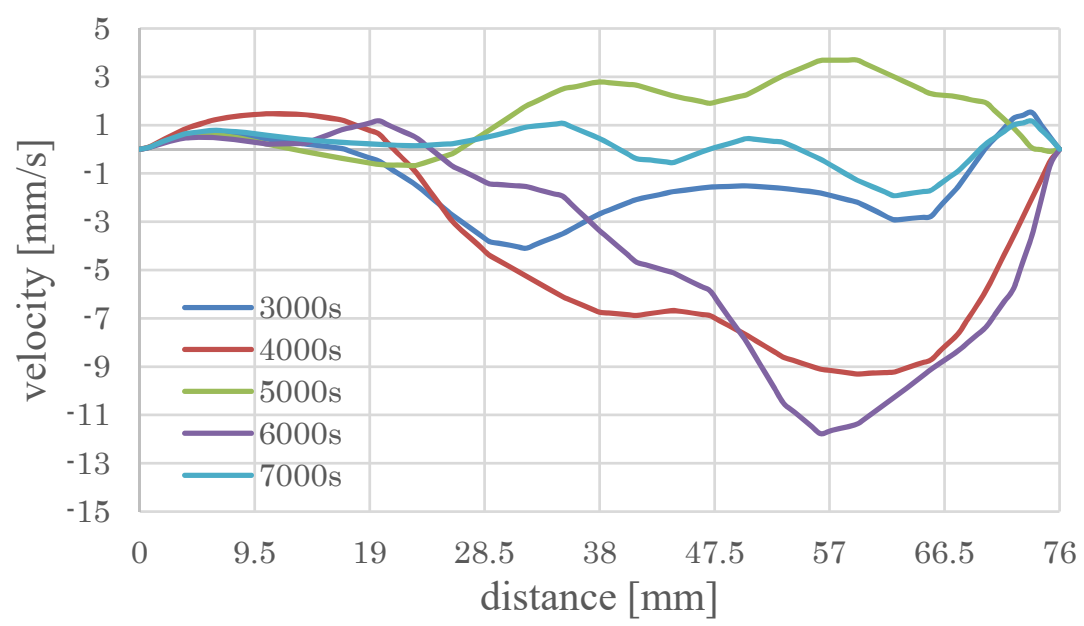

Figure 8: Gravity direction velocity profile of centre line at different times.

\section{Conclusions}

A numerical simulation of flow behaviour in the glass melter, which is used in the HLLW verification, was conducted by GSMAC-FEM method in this study. In the glass melter, the flow is occurred by Joule-heating and affected by flow field, thermal field and electro-magnetic field. In the previous CFD simulation, the effect from magnate filed can't be calculated. GSMAC-FEM method can execute full coupling analyses of flow field, thermal field, and electromagnetic field. To know flow behaviour in the glass melter more obviously, GSMAC method was used to calculate the flow behaviour in a cuboid cavity which modelled the upper part of the glass melter. Flow behaviour under adiabatic cases and cooling case of the electrode cooling condition was calculated by the GSMAC method. Conclusions are shown as follows.

(1) The temperature change with time in the centre point of the cavity was similar under 2 cases. The growth rates of temperature of 2 cases were same. However, because the stable temperature of cooling case was lower than the adiabatic case, the electrodes cooling case became stable was faster than electrodes was adiabatic.

(2) In the cuboid cavity, the Joule-heating flow occurred in the whole of the cavity. However, when the electrodes are under cooling condition, the stable downflows occurred near the electrodes side. And the counter flow of these stable downflows made flow in the bottom of the cavity was stable.

(3) Under the cooling electrodes condition like glass melter, the distance of downflow which can be achieve was a little shorter than adiabatic condition. However, the chaotic flow could be observed in over $80 \%$ upper part of the cavity. The vitrification of HLLW will not be weakened. Cooling electrodes in the glass melter just protect the electrodes. 
The Joule-heating flow in the cuboid bottom cavity was observed by the GSMA-FEM code. The flow in the glass melter can be predicted by this code. The chaotic flow occurred in the whole upper part of the glass melter. In addition, cooling electrodes in the glass melter just protect the electrodes, the chaotic flow also occurred almost in the whole upper part of the glass melter. Therefore, the effect of the electrodes cooling condition needn't be considered at glass melter design.

\section{References}

[1] N. Tsuzuki, H. Kikura, H. Kawai, T. Kawaguchi, A. Iganaki, E. Ochi, "The numerical analysis on unsteady flow in the cavity whit Joule-heating," 48th Heat Transfer Symposium of Japan, Okayama, Japan, 2011-6, Volume III, pp. 727-728 (2011).

[2] N. Tsuzuki, H. Kikura, Y. Saito, H. Kawai, G. Hashimoto, K. Minami, E. Ochi, "Changes of flow behaviour due to magnetic permeability of the fluid in the Joule heating field," Thermal Engineering Conference 2011, Shizuoka, Japan, 2011-10, No. 11-36, pp. 283-284 (2011).

[3] T.T. Duong, H. Tanaka, N. Tsuzuki, H. Kawai, H. Kikura, "Effect of cooling temperature of electrodes on Joule-heating flow in cubic cavity," Progress in Nuclear Energy. Volume 82, July 2015, pp. 165-175.

[4] T.T. Duong, H. Tanaka, N. Tsuzuki, H. Kawai, H. Kikura, "Experimental and Numerical Investigation of Joule-heating flow in a Square Cavity Effect of Cold Cap Condition," International Topical Meeting on Advances in Thermal Hydraulics - 2014, Reno, Nevada, Los Angeles, America, 2014-6, CD-ROM, Paper 10300, pp. 1-6 (2014).

[5] H. Igarashi, K. Kawamura, T. Takahashi, "Effect of noble metal elements on viscosity and electrical resistivity of simulated vitrified products for high-level liquid waste," MRS Online Proceedings Library, Volume 257, p. 169 (1991).

[6] K. Oda, M. Kaminoyama, "Mathematical Modeling of Sulfate Fining in a Glass Melting Furnace," Journal of Chemical Engineering of Japan, Vol. 42, No. 12, pp. 868-877 (2009).

[7] A. Aoshima, "The technology development for long life vitrification melting furnace," 2009.

[8] T. Tanahashi, H. Okanaga, T. Saito, "GSMAC Finite element method for unsteady incompressible Navier-Stokes equation at high Reynolds number", International Journal for Numerical Method, 11 (1990), pp. 479 499.

[9] C.H. Jung, T. Tanahashi, and Y. Ogawa, "GSMAC-FEM analysis of single crystal growth by Cusp MCZ method", KSME International Journal, 15, (2001), pp. 1876-1881.

[10] H. Kohno, T. Tanahashi, "An application of GSMAC-FEM to coupled natural and Marangoni convection in a square cavity", International Journal of Computational Fluid Dynamics, 19, No. 4 (2005), pp. 329-335. 